\title{
A concept for multiterawatt fibre lasers based on coherent pulse stacking in passive cavities
}

\author{
Sven Breitkopf ${ }^{1}$, Tino Eidam ${ }^{1,2}$, Arno Klenke ${ }^{1,2}$, Lorenz von Grafenstein ${ }^{1}$, Henning Carstens ${ }^{3,4}$, \\ Simon Holzberger ${ }^{3,4}$, Ernst Fill ${ }^{3,4}$, Thomas Schreiber ${ }^{5}$, Ferenc Krausz ${ }^{3,4}$, Andreas Tünnermann ${ }^{1,2,5}$, \\ Ioachim Pupeza $^{3,4}$ and Jens Limpert ${ }^{1,2,5}$
}

Since the advent of femtosecond lasers, performance improvements have constantly impacted on existing applications and enabled novel applications. However, one performance feature bearing the potential of a quantum leap for high-field applications is still not available: the simultaneous emission of extremely high peak and average powers. Emerging applications such as laser particle acceleration require exactly this performance regime and, therefore, challenge laser technology at large. On the one hand, canonical bulk systems can provide pulse peak powers in the multi-terawatt to petawatt range, while on the other hand, advanced solid-state-laser concepts such as the thin disk, slab or fibre are well known for their high efficiency and their ability to emit high average powers in the kilowatt range with excellent beam quality. In this contribution, a compact laser system capable of simultaneously providing high peak and average powers with high wall-plug efficiency is proposed and analysed. The concept is based on the temporal coherent combination (pulse stacking) of a pulse train emitted from a high-repetition-rate femtosecond laser system in a passive enhancement cavity. Thus, the pulse energy is increased at the cost of the repetition rate while almost preserving the average power. The concept relies on a fast switching element for dumping the enhanced pulse out of the cavity. The switch constitutes the key challenge of our proposal. Addressing this challenge could, for the first time, allow the highly efficient dumping of joule-class pulses at megawatt average power levels and lead to unprecedented laser parameters.

Light: Science \& Applications (2014) 3, e211; doi:10.1038/Isa.2014.92; published online 10 October 2014

Keywords: cavity dumping; cavity enhancement; coherent pulse addition; high average power and high peak power; ultrafast lasers

\section{INTRODUCTION}

The scope of any laser application crucially depends on the quality of the driving light source, i.e., the laser itself. Hence, it is not surprising that improved laser output parameters have repeatedly extended the range of applications or even opened up novel opportunities. ${ }^{1,2}$ Today, ultrafast laser systems are considered as unique tools for many industrial and scientific applications, the latter one being an incubator for seminal discoveries and new technologies. One of these emerging applications is laser-wakefield particle acceleration. Here, an extremely intense femtosecond laser pulse that propagates in a plasma can be employed to accelerate particles, such as electrons, in its wakefield. ${ }^{3,4}$ There is a growing interest in laser-wakefield particle acceleration schemes due to the fact that classical radiofrequency-driven machines are about to reach their intrinsic limitations given by their acceleration gradient and, thus, size and cost. ${ }^{5}$

However, even near-future accelerator applications will require parameters that are beyond the ones that can be achieved with stateof-the-art ultrafast lasers, particularly in terms of average power. To achieve $\mathrm{TeV}$-level energies for electrons and positrons via laser plasma acceleration with fluxes comparable to those of radiofrequency accelerators, a laser architecture that efficiently allows for the combination of petawatt peak powers with megawatt average powers is required. For example, Leeman et al. ${ }^{6}$ estimated that at a laser wavelength of around 1 $\mu \mathrm{m}$, a pulse energy of $32 \mathrm{~J}$ at a repetition rate as high as $15 \mathrm{kHz}$ is required. This corresponds to an average power of $480 \mathrm{~kW}$, combined with pulse durations as short as possible (preferably sub-100 fs) and an almost diffraction limited beam quality. Once these parameters are reached, about 100 of those stages are needed to enable a compact $\mathrm{TeV}$-scale accelerator facility. This parameter range is far beyond the capability of any existing laser technology today. Hence, developing such a laser system is an extremely challenging task. Such a development might well take several decades of aggressive research and development, and, most importantly, novel concepts and approaches.

Current state-of-the-art femtosecond solid-state lasers, such as titanium-sapphire-based amplification systems, can generate high peak intensities. In terms of peak power, one benchmark laser system currently is BELLA ${ }^{7}$ producing 30 -fs pulses with $40 \mathrm{~J}$ of pulse energy, i.e. $>1 \mathrm{PW}$ peak power, at a repetition rate of $1 \mathrm{~Hz}$ resulting in an average

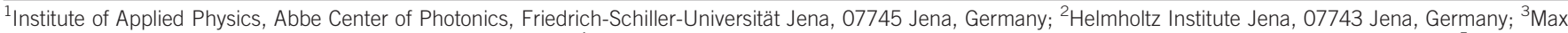

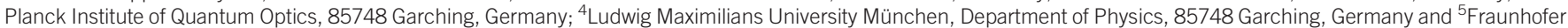
Institute for Applied Optics and Precision Engineering, 07745 Jena, Germany

Correspondence: S Breitkopf, Friedrich-Schiller-Universität Jena, Institute of Applied Physics, Albert-Einstein-Straße 15, 07745 Jena, Germany

E-mail: sven.breitkopf@uni-jena.de

Received 11 April 2014; revised 23 July 2014; accepted 24 July 2014 
power of $40 \mathrm{~W}$. Still, BELLA is far from being compact or efficient with a wall-plug efficiency in the range of $10^{-4}$. Taking into account the envisaged performance and number of stages mentioned above, such a poor efficiency and high costs would be a roadblock for laser-based particle accelerators. Moreover, a scaling of the average power and, hence, of the repetition rate, is inhibited by thermo-optical problems such as thermal lensing and stress-induced birefringence in the amplifying medium. Thus, a PW-class laser with a repetition rate of $1 \mathrm{~Hz}$ (corresponding to a few 10s of Watts of average power) constitutes the frontier of laser technology today. This performance has opened the door to a vast range of scientific opportunities, including proof-ofprinciple experiments on the most advanced accelerator concepts, among them plasma-channel waveguide-assisted wake-field accelerators that enable enormous accelerating gradients and mono-energetic particles. ${ }^{7}$ However, only a higher average power and a high-repetitionrate driving laser source, respectively, can bring these laboratory-tested concepts to a facility which benefits a broad range of users and their industrial and medical applications. This requirement leads to a major challenge lying in the fact that scaling the peak and the average power of a laser are two completely different tasks, which usually impose contradictory requirements on the geometry of the active medium.

Over the past decades, solid-state-laser concepts with advanced active medium geometries have been developed, including the thin disk, ${ }^{8}$ the slab ${ }^{9}$ or the fibre. ${ }^{10}$ All of them feature an improved thermal management, which has enabled scaling the average power while preserving an excellent spatial beam quality. In particular, fibre-based amplifiers are well known for their outstanding beam quality, high efficiency and high average powers even in the femtosecond-pulse regime. ${ }^{11}$ However, fibres face serious challenges when to be pushed to high peak powers. The tight transverse confinement of the optical pulses over considerable lengths typically enforces nonlinear pulse distortions and damage. Thus, to generate high pulse energies and, consequently, peak powers, fibres with large mode-field areas are employed. ${ }^{12}$ Moreover, the technique of chirped-pulse amplification (CPA) is essential to stretch the laser pulses in time and, hence, reduce their peak power during amplification. ${ }^{13}$ State-of-the-art grating-based stretchers lengthen femtosecond pulses up to several nanoseconds and have allowed fibre-based systems to produce pulse peak powers of up to $3.8 \mathrm{GW} .{ }^{14}$ In principle, the stretched pulse duration could be further increased but in practice this parameter is limited to about $10 \mathrm{~ns}$ by the dimensions of the laser system and the available grating sizes.

Parallelisation offers an intuitive approach of overcoming the fundamental limitations of a single amplifier channel. Here, the basic idea is the coherent combination of multiple emitters to reach peak and average powers not achievable with single-aperture systems. ${ }^{15}$ This technique has been first applied to continuous-wave lasers ${ }^{16}$ and more recently extended to ultrafast systems. ${ }^{17-19}$ This is a particularly interesting approach for fibre amplifiers, since their high gain and compact single-pass set-ups allow for straight-forward parallelisation. Parallel amplification can be regarded as an artificial increase of the mode-field diameter, which, therefore, enables the scaling of both the pulse peak power and the average power by a factor roughly equal to the number of amplification channels. So far, in low-power operation up to 64 passive channels have been combined, ${ }^{20}$ whereas in high-power operation the state of the art is a set-up combining the output of four femtosecond amplifiers. ${ }^{21}$ With this system $530 \mathrm{~W}$ of average power and a pulse energy of $1.3 \mathrm{~mJ}$ have been realized with combining efficiencies exceeding 93\%. Furthermore, it has also been theoretically shown ${ }^{22,23}$ that the efficiency can be kept high even up to more than thousand channels. These promising experimental results have encouraged research into particle acceleration based on the coherent combination of a large number (possibly millions) of fibre amplifiers. ${ }^{24}$ In this concept, each fibre amplifier would have to generate pulses with energies in the $\mathrm{mJ}$ range at a $\mathrm{kHz}$ repetition rate. As a consequence, they would have to be operated at average power levels as low as a few watts. This means that the fibres would be underperforming in terms of average power leaving their essential advantage unexploited. Additionally, due to the immense number of required optical elements, the complexity and the cost of such an approach would be far beyond any tolerable value.

A possible solution for this problem is the temporal combination of several pulses, a technique that has been successfully shown in socalled divided-pulse-amplification (DPA) systems. ${ }^{25-27}$ Here, each pulse is split in time into a train of pulses via birefringent crystals or via a combination of beam splitters and free-space delay lines. Subsequently, the individual lower-intensity pulses are amplified and, finally, coherently temporally combined. This method can be considered as an effective increase of the stretched pulse duration and thereby, correspondingly, of the compressible pulse energy beyond the limits imposed by conventional stretchers. Moreover, DPA can also be implemented in combination with the spatially separated amplification discussed above. Thus, in the ideal case, with DPA, the output pulse energy emitted by a single fibre amplifier can be increased according to the number of pulse replicas. Therewith, DPA is a promising way to scale the pulse energy by about one order of magnitude. However, increasing the number of pulse replicas to 100 or even 1000 with traditional DPA approaches in the CPA regime and, thus, achieving TW-peak powers with fibre amplifiers will become extremely challenging due to the required number and length of the delay lines, their individual stabilisation and the necessary mitigation of saturation effects in the amplifier. ${ }^{26}$ Hence, an interesting alternative to DPA is to start with a higher repetition rate and to use only one delay line for stacking hundreds or even thousands of pulses. Such a delay line can be realized in the form of a passive enhancement cavity. In this paper, we analyse the concept of stacking stretched pulses of a high-repetition-rate, high-average-power femtosecond CPA system in a passive cavity. After a certain number of pulses, i.e., one stacking period, a fast switching element is used to couple out the intense circulating pulse, which is then compressed back to its fs-duration. Although the use of cavity-dumped enhanced pulses has already been demonstrated in the past for low-power systems, ${ }^{28,29}$ the combination of today's femtosecond laser systems together with state-of-the-art enhancement cavities ${ }^{30}$ can result in a new class of laser output parameters vastly outperforming anything demonstrated before. In general, this 'stack-and-dump' ( $\mathrm{SnD}$ ) technique can be applied to any existing amplifier technology. However, it is particularly well suited for amplifier geometries that can deliver very high average powers such as rare earth-doped fibres.

\section{MATERIALS AND METHODS}

Ultrashort-pulse enhancement cavities have already shown their huge potential for stacking laser pulses over the last decade. Such cavities are simple mirror arrangements without any transmissive elements and, therefore, ideally suited for handling highest laser powers. Recently, with an enhancement factor of 2000, 10 ps pulses were enhanced to $670 \mathrm{~kW}$ of intracavity average power. ${ }^{31}$ So far, these systems are mainly used for the generation of short-wavelength radiation via intracavity high-harmonic generation ${ }^{31-34}$ or inverse Compton scattering. ${ }^{35}$

The SnD concept employs an enhancement cavity for coherent pulse stacking. The main difference, however, is that the pulses are 
coupled out of the cavity after a certain number of round-trips before the steady state is reached. Potential intensity-related limitations are mitigated due to the long pulse durations of the stretched CPA pulses, which, in principle, should allow for much higher pulse energies in a loaded cavity than fs- or ps-pulses did in the past. ${ }^{30} \mathrm{~A}$ schematic drawing of the basic concept is depicted in Figure 1a. For their temporal stacking, the pulses are coupled into a cavity with a length corresponding to the repetition rate $f_{\text {rep }}$ of the incident pulse train, which is typically on the order of $10 \mathrm{MHz}$. Initially, there is no field inside of the cavity and, therefore, only a small fraction of the first pulse (given by the transmittivity $T$ of the input-coupling mirror) is coupled in. When the cavity roundtrip time is matched to the repetition rate of the incident pulses, coherent build-up inside the cavity is ensured. In this way, the pulse energy stored in the cavity increases with every round trip. Steady-state is reached when the energy coupled to the cavity balances exactly the roundtrip losses $L$. Typically, a power enhancement of a few orders of magnitude can be achieved. ${ }^{30,36}$ However, before this state is reached, in the $\mathrm{SnD}$ concept the intracavity pulse is coupled out via a switch faster than $1 / f_{\text {rep }}$ and at a switching rate $f_{\text {switch }}$ before the next stacking period begins, i.e., the cavity is operated in a non-steady-state regime.

The main challenge of the proposed approach lies in the dumping of the enhanced pulse. On the one hand, the cavity should be as long as possible in order to provide a sufficiently long time window for the switch. On the other hand, for practical reasons (number of required mirrors, stability, foot-print of the system) the cavity should be as short as possible. A practical tradeoff is a cavity length of $30 \mathrm{~m}$ corresponding to a repetition rate of $10 \mathrm{MHz}$ and a required switching time of 100 ns between two successive pulses. Such a length can, if folded, still be operated on one optical table and these values are assumed in the following calculations. In order to achieve an output repetition rate of e.g., $f_{\text {switch }}=15 \mathrm{kHz}, N=666$ pulses have to be stacked within one stacking period according to $f_{\text {switch }}=f_{\text {rep }} / N$.

For a sufficiently high overall wall-plug efficiency of the proposed system, a high stacking efficiency $\eta_{\text {cav }}$ is crucial. This parameter is defined as the circulating energy of the enhanced pulse divided by the sum of the energy of the input pulses over one complete stacking period. The output pulse energy $E_{\text {out }}$ of a non-steady-state enhancement cavity is given by

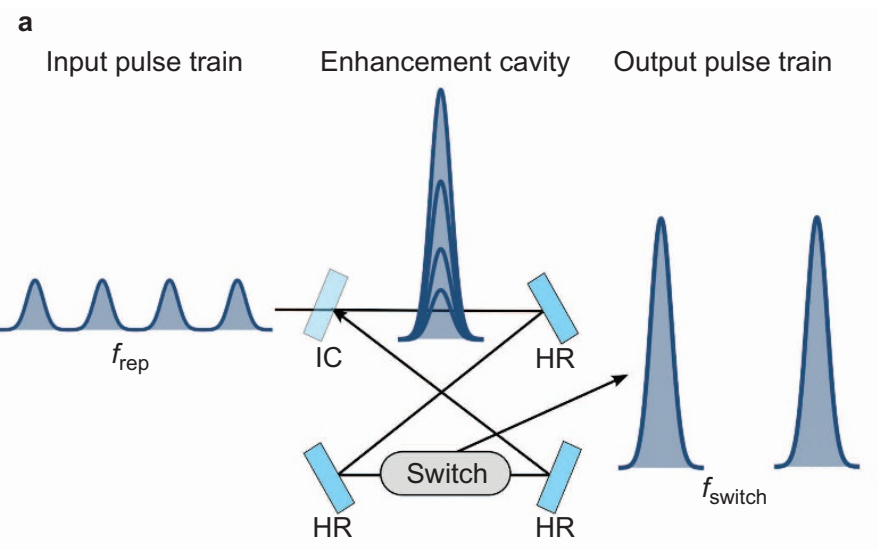

$$
E_{\text {out }}=E_{\text {in }} N \eta_{\text {cav }} \eta_{\text {switch }}
$$

where $N$ denotes the number of input pulses with energy $E_{\text {in }}$ over one stacking period and $\eta_{\text {switch }}$ is the output-coupling efficiency of the switch. The stacking efficiency of the cavity $\eta_{\text {cav }}$ is determined by the round-trip losses $L$ (this comprises all losses inside the cavity except for the transmission losses at the input-coupler) and the loading losses, i.e., the amount of energy reflected at the input port during the build-up. The stacking efficiency $\eta_{\text {cav }}$ can be further improved by shaping the amplitude of the incident pulse trains, as discussed later. Figure 2 depicts the calculated stacking efficiency $\eta_{\text {cav }}$ as a function of the input-coupler transmittance $T$ for different cavity round-trip losses $L$. The graphs have been derived assuming perfect phase stabilization, i.e., possible decreases in efficiency due to residual phase fluctuations are neglected. There is a clear optimum for the input coupler transmittance $T$. For values of $T$ smaller than this optimum value, the loading losses are dominant, while for values of $T$ higher than the optimum, the round-trip losses dominate. Analogously to the steady-state case, the optimum transmittance also increases for higher losses.

It can be seen from Figure 2 that the round-trip losses have to be well below $0.1 \%$ in order to stack 666 pulses within the cavity without causing a critical reduction of the wall-plug efficiency due to cavity losses. This strict demand on $L$ could be relaxed by employing a nonuniform input pulse train of increasing amplitude within a stacking period. Two possible pulse-train shapes are depicted in Figure 3a. A stacking period of constant pulse energy (orange curve) and exemplary a slow exponential growth (blue dashed curve) are considered in the following. The efficiency improvement that can be achieved by using a pulse train with growing amplitude (Figure $3 \mathrm{~b}$ ) results from two effects. First, a part of the total input energy is shifted towards the end of the stacking period where the effective input-coupling efficiency of each pulse (defined by the intracavity field and $T$ ) is higher. Since this energy shift decreases the impact of the loading losses, a higher $T$ value can be chosen compared to the case of constant amplitude, which additionally increases the efficiency. Generally, the efficiency gets better for a stronger growth of the pulse train. However, typically a tradeoff between the stacking efficiency and the restrictions from the input-signal amplifiers has to be found.

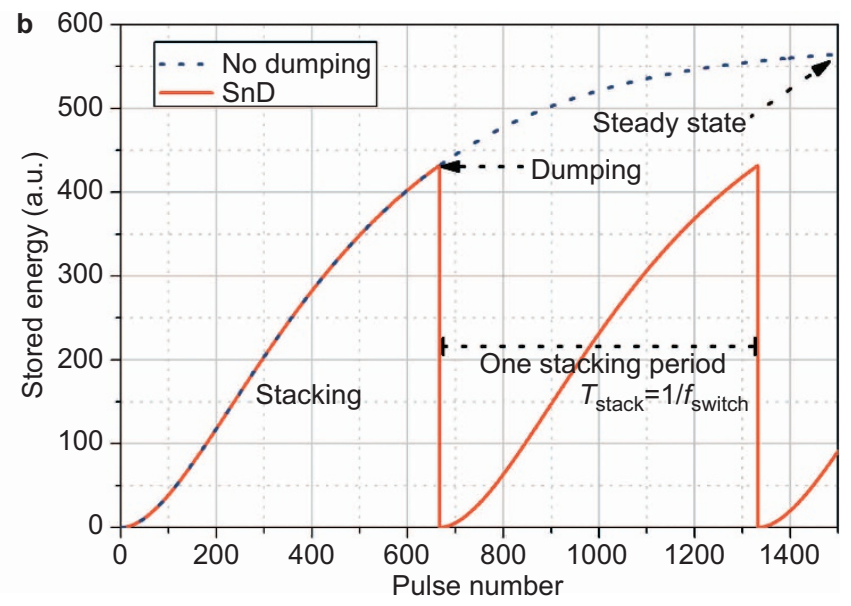

Figure 1 (a) Basic principle of a stack-and-dump enhancement cavity consisting of one input coupling mirror (IC) and three high reflective mirrors (HRs). The pulses are continuously coupled to the cavity with a repetition rate $f_{\text {rep }}$ leading to a high-energy intracavity pulse. After a certain number of incident pulses, the intracavity pulse is coupled out via a fast switch operating at a rate $f_{\text {switch. }}$ (b) In this schematic, the stored energy within the cavity in dependence on the incoming pulse number is depicted for the steady-state operation as well as for the SnD case. One stacking period is defined as $T_{\text {stack }}=1 / f_{\text {switch. }}$ SnD, stack-and-dump. 


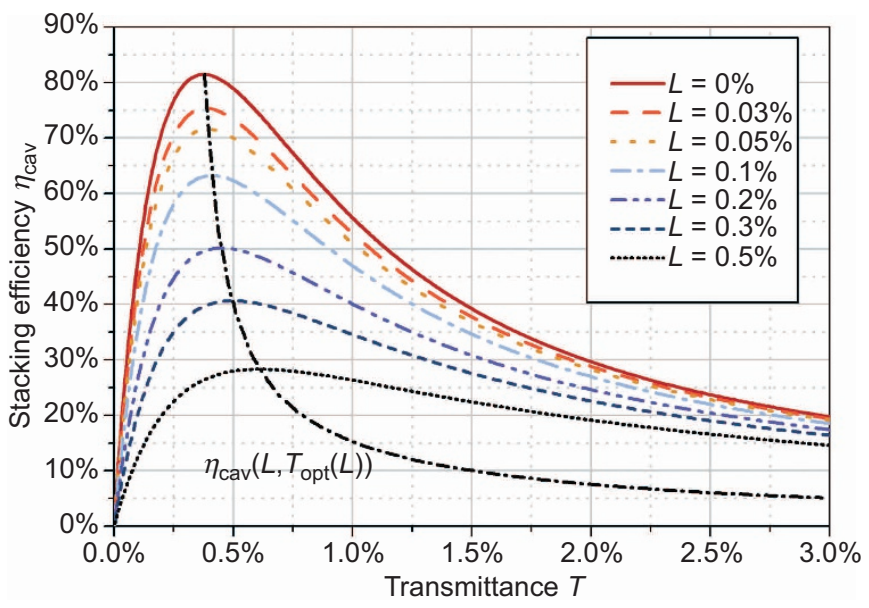

Figure 2 Calculated stacking efficiency as a function of the transmittance of the input coupling mirror for different cavity round-trip losses $L$ and 666 pulses per stacking period. The dashed-dotted black line depicts the highest possible stacking efficiency achievable with certain losses $L$ (implying an optimum value for $T$ ).

Figure $3 \mathrm{~b}$ shows the achievable stacking efficiency for these two shapes for different roundtrip losses $L$. It can be seen that, for example, for the slow exponential growth, a loss of $L=0.1 \%$ instead of $0.03 \%$
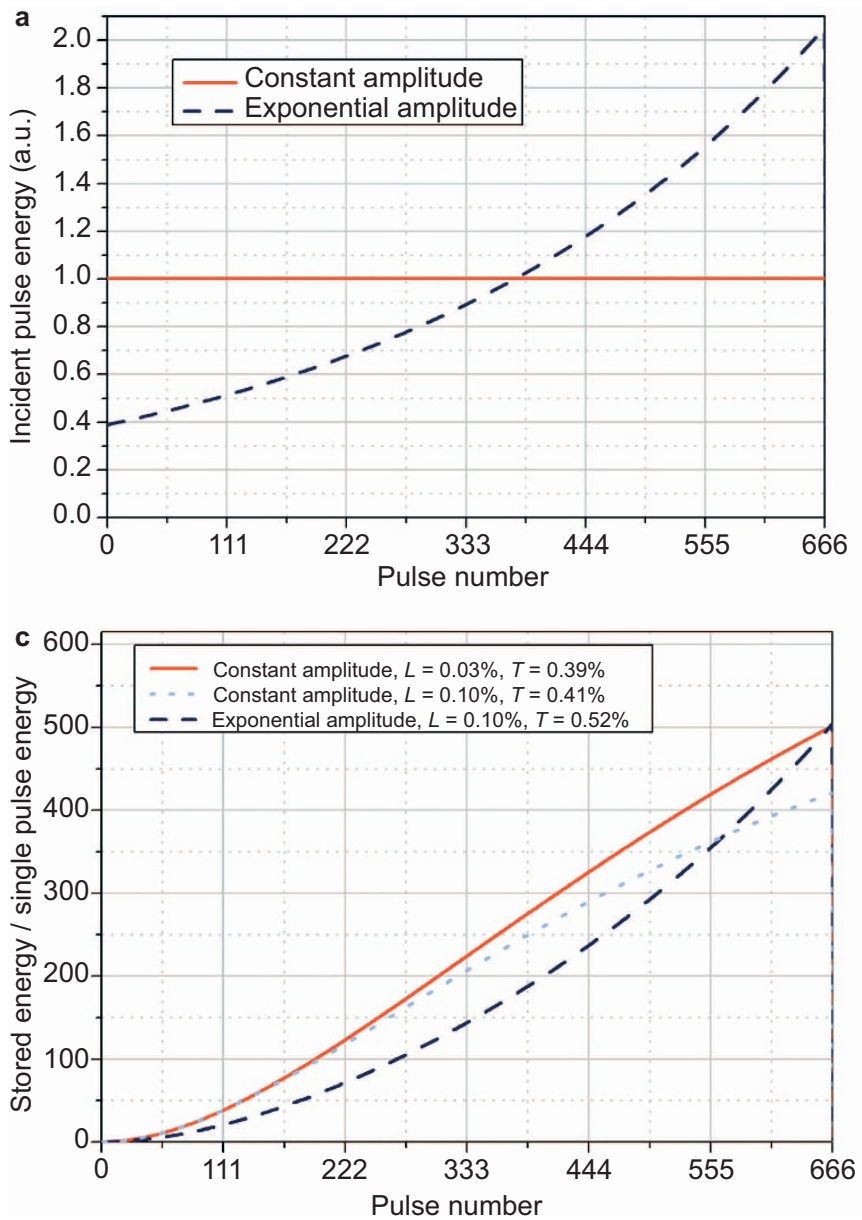

can be tolerated in order to realize a $75 \%$ stacking efficiency. Commercially available mirror coatings have reflectivities of 99.997\% and even higher. Furthermore, the cavity has to be placed in a vacuum chamber so there are no further losses introduced by scattering or absorption from air molecules. As a consequence of these facts, even the use of 10 mirrors would imply losses as low as $0.03 \%$ and with improved coating technology the losses will decrease even further.

Figure $3 c$ shows the evolution of the stored energy with respect to the number of incoming pulses. Two unshaped cases with different losses (orange and cyan curves) and one case for the exponentially shaped amplitude evolution (black curve) according to Figure la are depicted. In each case, the input-coupler transmittance $T$ is chosen in order to maximize $\eta_{\text {cav. }}$ It can be seen that, for the same losses of $0.1 \%$, the growth in stored energy is lower for the shaped pulse train at the beginning of the stacking period. However, as already discussed above, this is overcompensated at the end of the stacking period resulting in a total stored energy (and efficiency) equal to the depicted case of a constant pulse train with only $0.03 \%$ losses. Additionally, Figure $3 \mathrm{~d}$ shows the evolution of the input-coupling efficiency, which is the ratio of pulse energy coupled in the cavity compared to the energy of the incident pulse. Contrary to the steady-state case, the inputcoupling efficiency possesses a maximum at an intermediate pulse number.
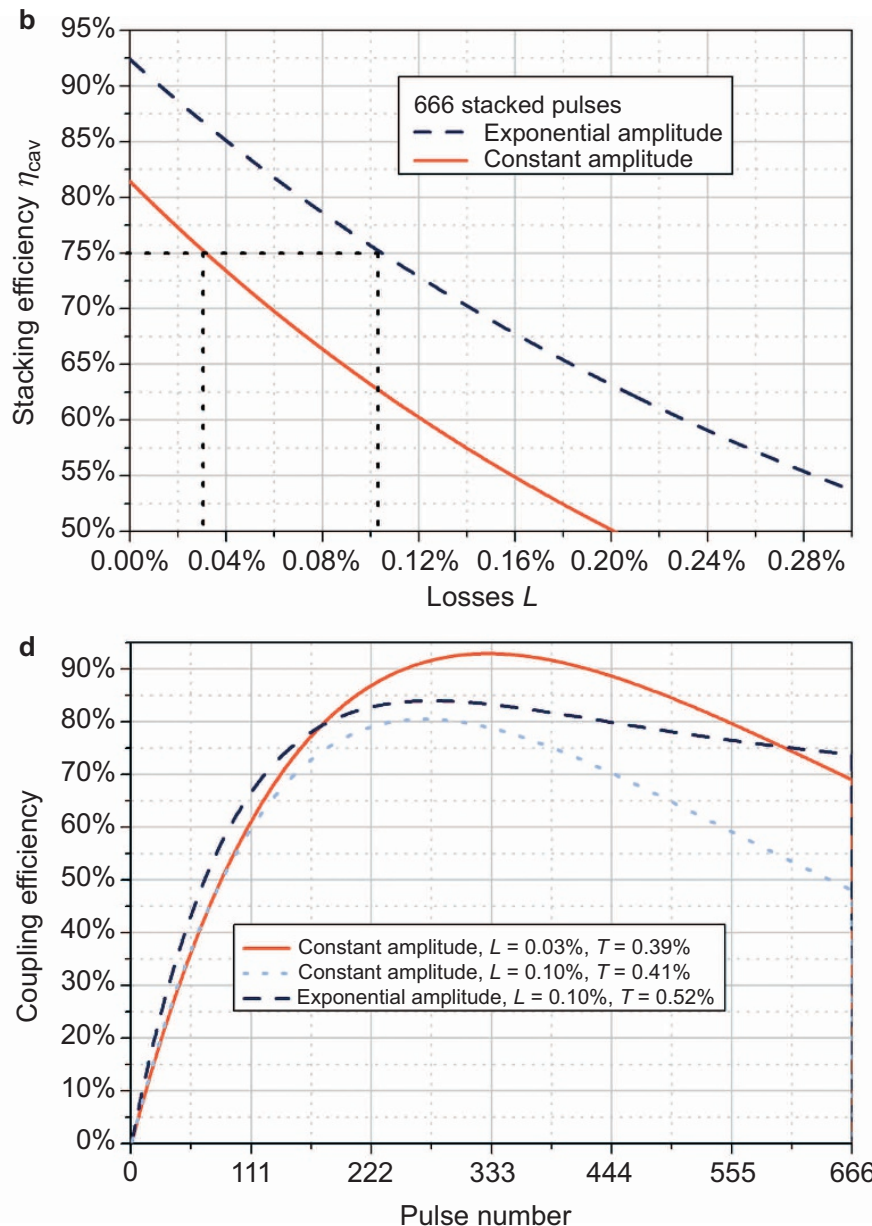

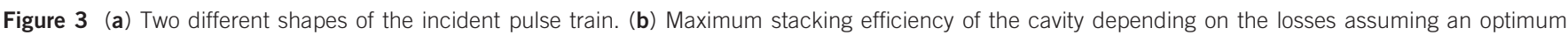

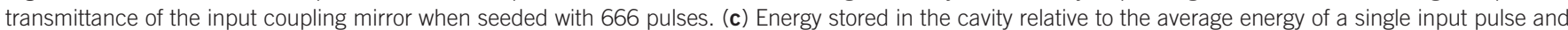
(d) development of the coupling efficiency as a function of the pulse number in the input pulse train. 
To dump the enhanced pulse out of the cavity, a switchable element has to be implemented in the passive resonator. In order to keep the roundtrip losses below $0.03 \%$, any additional loss due to this element has to be avoided. This implies that standard components such as Pockels cells or acousto-optic modulators cannot be employed since they are transmissive and typically introduce losses in excess of $0.1 \%$. Moreover, the circulating average power within the cavity would lead to damage of these elements. Hence, a purely reflective switch with less than 100 ns switching time (assuming a $10 \mathrm{MHz}$ cavity) is necessary to efficiently couple out the intracavity pulse. So far, several switching techniques have been considered and evaluated. For example, fast tilting cavity mirrors driven by piezo actuators ${ }^{37}$ or electro-optic deflectors could be employed to switch the beam out of the cavity. Additionally, interferometric output coupling employing a reflective grating as the splitting and combining element ${ }^{38}$ might be possible as well. At the moment, the most promising concept is a chopper wheel, similar to those used for energy or polarisation selection in particle beams. ${ }^{39}$ The wheel envisaged has mirror segments attached to its surface and rotates in the vacuum chamber next to the cavity beam. For 665 round trips, while the enhanced pulse builds up in the cavity, the wheel does not interact with the beam. However, during the 666th round trip, the pulse is coupled out by the mirror attached to the wheel, which crosses the optical path of the beam. A schematic setup of this switch is depicted in Figure 4a. In the case of a $10 \mathrm{MHz}$ input pulse train and $15 \mathrm{kHz}$ output repetition rate, 15 mirror segments attached to one chopper wheel in combination with a rotation frequency of $1 \mathrm{kHz}$ can fulfil the task of coupling out the enhanced pulses in a purely reflective manner. For a realistic chopper diameter of $30 \mathrm{~cm}$, the reflecting area has to be very narrow (about $100 \mu \mathrm{m}$ ) in order to interact only during the 666th round trip without disturbing the previous one. Fortunately, such thin mirrors are actually not necessary. If the mirror is broader and blocks the subsequent pulses, either a specially designed burst-mode laser input ${ }^{40}$ could be employed or the first few pulses in each stacking period are discarded resulting only in a slight reduction of the efficiency. Moreover, the beam width $d_{1}$ at the chopper wheel has to be $<100 \mu \mathrm{m}$ in order to guarantee a complete overlap with the rotating mirror. Damage issues can be avoided by shaping the beam elliptically at this $z$-position in the cavity and by using the mirror under grazing incidence, i.e., the small beam diameter in the $x$-direction is projected on the large mirror surface (Figure $4 \mathrm{~b}$ ). Similarly to the cavity and the laser oscillator, the rotation frequency of the chopper wheel has to be stabilized and synchronized to an external clock. Additionally, due to their high mass and the corresponding moment of inertia as well as their purely magnetic mounting, state-of-the-art chopper wheels typically possess only a slow timing jitter and a high stability of the rotation axis guaranteeing stable output-beam parameters (energy and pointing). The pointing stability is limited by the positioning of the mirror segments on the chopper wheel. Any larger deviations therefore reoccur periodically at $15 \mathrm{kHz}$ and hence can and will be stabilized by an active stabilisation mechanism that can be readily implemented after the output of the cavity. The mechanical vibrations introduced by the chopper wheel to the system are expected to be comparable to those of turbomolecular vacuum pumps employed in standard cavity enhancement set-ups and, thus, uncritical for the optical alignment.

As mentioned above, the average power circulating in the cavity will be orders of magnitude higher than the output average power. However, the cavity itself comprises exclusively reflective elements, which results in high damage thresholds and low thermal loads. Recently, with advanced cavity designs, an average power of up to $670 \mathrm{~kW}$ was demonstrated ${ }^{31}$ in a $2.4-\mathrm{m}$ long ultrashort-pulse enhancement cavity. In order to obtain high peak intensities in a focus, that cavity was operated close to a stability edge, ${ }^{41}$ where spatial modes can simultaneously become resonant due to approaching degeneracy and can be coupled by thermally induced aberrations. This effectively limits the achievable average power. ${ }^{31}$ As for $\mathrm{SnD}$ no high intensity in the cavity is required, this limitation can be circumvented by operating the cavity close to the stability centre. In Ref. 31, it was also shown that the thermal sensitivity decreases as the cavity length increases, making the 30-m long cavity well suited for average powers above $1 \mathrm{MW}$. Although the projected pulse energy is 1000 times higher compared to, ${ }^{32}$ the pulses are 16000 times longer, leading to a significantly lower peak power and correspondingly lower intensities on the cavity optics for comparable beam sizes. Thus, intensity related

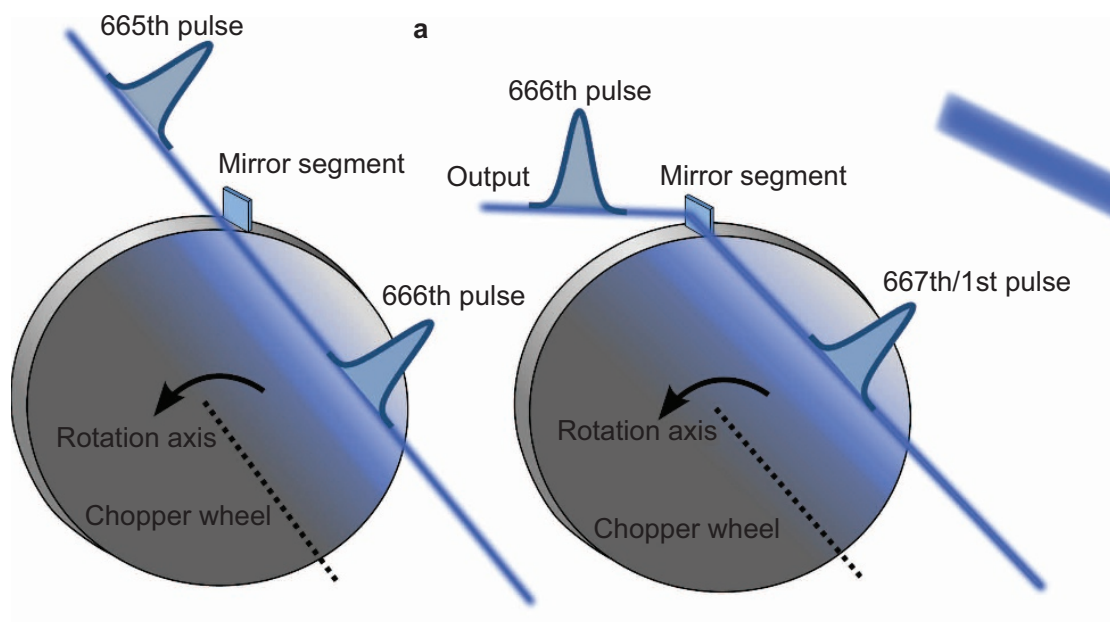

Rotation axis

b 


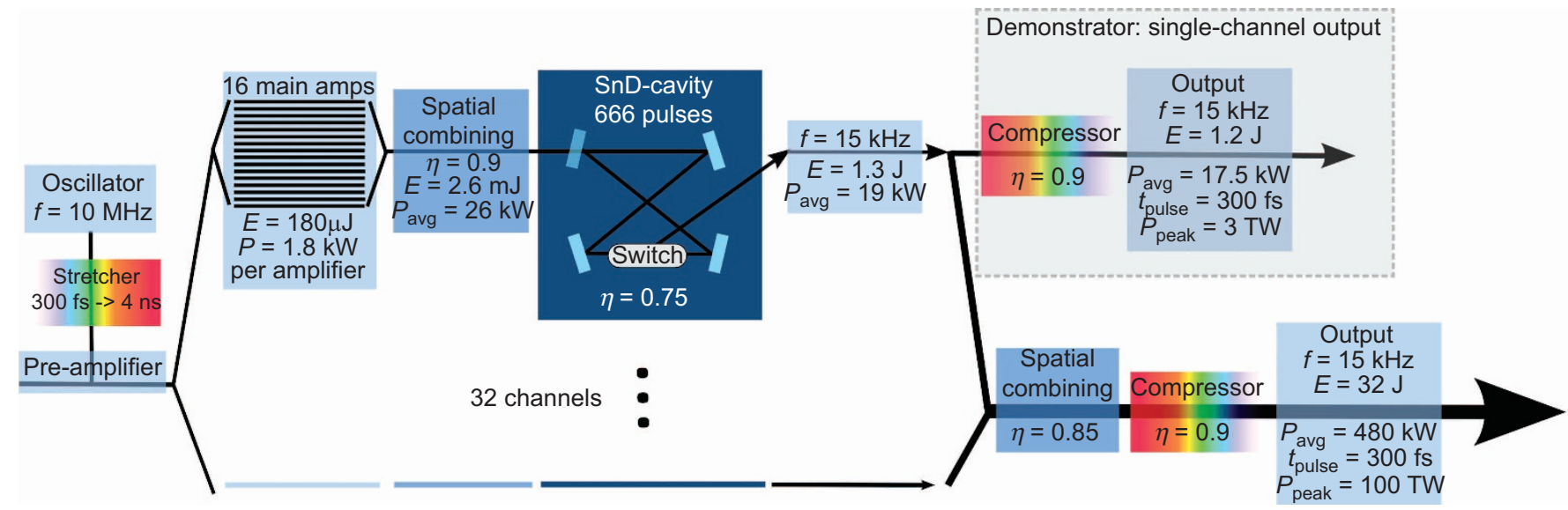

Figure 5 System design for achieving extremely high peak powers in the PW range at very high average powers in the MW-range employing spatial coherent combination and temporal stacking in an enhancement cavity.

limitations such as third-harmonic generation or damage are not to be expected.

There are some further advantages in employing $\mathrm{SnD}$ cavities as temporal combiners. Due to the resonator geometry the spatial mode quality of the beam is expected to be excellent and since the pulse is directly coupled out, the emitted beam should remain close to the beam profile within the cavity, which can be close to diffraction-limited even in high-power operation. ${ }^{41}$ Additionally, due to the nature of the reflective dumping of the pulses using the chopper wheel, there is no signal at the output port of the cavity between pulses. Hence, there will be no background noise before or after the time frame given by the mirror size.

\section{RESULTS AND DISCUSSION}

The $\mathrm{SnD}$ concept described so far is most suitable for implementation in conjunction with state-of-the-art fibre CPA systems. Even though a realisation with other amplifier geometries might be possible, fibre amplifiers are ideal candidates for this technique since they possess high wall-plug efficiencies, excellent beam qualities and can easily be parallelized due to their simple single-pass amplification set-up. Thus, in the following, a concept of such a fibre-based multi-TW system will be sketched. As depicted in Figure 5, the pulses delivered by a $10-\mathrm{MHz}$ oscillator are stretched from 300 fs to $4 \mathrm{~ns}$. Afterwards, they are preamplified and split into 16 equal main-amplifier fibres. Each of these fibres is assumed to emit $180 \mu \mathrm{J}$ pulse energy at $1.8 \mathrm{~kW}$ average power, which is a set of parameters that is very likely to be achieved in the near future with the next generation of optical fibres. These parallel channels are recombined with an efficiency of $90 \%{ }^{21}$ and sent into a 30-m long enhancement cavity. The enhanced pulses are dumped at $15 \mathrm{kHz}$ using the $\mathrm{SnD}$ technique. As shown earlier, the efficiency of the cavity can be estimated to be at least $75 \%$. The pulse energy attainable from only 16 fibres at this stage of the set-up after a compression stage with $90 \%$ efficiency can already be as high as 1.2 J. Considering the repetition rate of $15 \mathrm{kHz}$, this is a parameter set that could be readily used for many demanding applications.

In order to achieve even better output parameters, further parallelisation can be implemented. For the target parameters envisaged for particle-acceleration applications, 32 of these output channels could be again coherently combined before compression. Assuming e.g. a combining efficiency of $85 \%$, the pulse energy at $15 \mathrm{kHz}$ repetition rate is expected to be as high as $32 \mathrm{~J}$.
An interesting variation of the approach depicted in Figure 5 is to also employ the cavity for spatial beam combining by using more than one input coupling mirror and hence reduce the number of cavities to only one. Although this would increase the peak and average power inside the cavity even more, this approach would significantly reduce the complexity of the proposed system.

\section{CONCLUSIONS}

Novel solid-state amplifiers and enhancement cavities, as well as the methods for coherent pulse combination, have reached a development point where they could benefit from each other in a manner allowing for the efficient generation of an entirely new class of laser output parameters. Here, we present a concept that aims at transforming the high repetition rate of a front-end femtosecond laser system to a lower value and, thereby, at increasing the output pulse energies by almost the same factor without a significant loss of average power. This approach could combine extremely high peak powers (up to the PW level) and average powers (approaching $1 \mathrm{MW}$ ). These parameters would benefit numerous applications. In particular, laser-wakefield acceleration could be made possible in facilities comparable, if not superior, to standard RF-based particle accelerators in terms of price, size and efficiency. For a target laser system with $32 \mathrm{~J}$ of pulse energy and $15-\mathrm{kHz}$ repetition rate, the proposed design concept could reduce the required number of channels from $10^{5}-10^{6}$ as in the approach employing only parallel amplification ${ }^{24}$ to 512 .

Experiments with a $10 \mathrm{MHz}$ cavity seeded by a state-of-the-art front-end fibre-CPA system will be carried out in the near future. Moreover, different switching technologies are currently being investigated and, in particular, the chopper wheel proposed here is under development.

\section{ACKNOWLEDGEMENTS}

This work has been partly supported by the German Federal Ministry of Education and Research (BMBF) under contract 13N12082 'NEXUS', by the Thuringian Ministry of Education, Science and Culture (TMBWK) under contract 12037-515 'BURST', by the European Research Council under the ERC grant agreement no. [617173] 'ACOPS' and by the Deutsche Forschungsgemeinschaft Cluster of Excellence 'Munich-Centre for Advanced Photonics' (munich-photonics.de). AK acknowledges financial support by the Helmholtz-Institute Jena. TE acknowledges financial support by the CarlZeiss-Stiftung. IP and $\mathrm{SH}$ acknowledge financial support by the BMBF under PhoNa-Photonische Nanomaterialien, contract number 03IS2101B. We 
acknowledge the support and fruitful discussions within the International Coherent Amplifier Network (ICAN) Consortium. We also acknowledge the support and fruitful discussions about the chopper wheel with Eberhard Rosenthal and Michael Butzek from the Forschungszentrum Jülich.

1 Ferray M, L'Huillier A, Li X. Multiple-harmonic conversion of $1064 \mathrm{~nm}$ radiation in rare gases. J Phys B 1988; 21: L31-L35.

2 Will I, Templin HI, Schreiber S, Sandner W. Photoinjector drive laser of the FLASH FEL. Opt Express 2011; 19: 23770-23781.

3 Tajima T, Dawson J. Laser electron accelerator. Phys Rev Lett 1979; 43: 267-270.

4 Hooker SM. Developments in laser-driven plasma accelerators. Nat Photonics 2013; 7: 775-782.

5 Döbert S. RF-breakdown in high-frequency accelerators. In: Proceedings of the IEEE International Power Modulator Conference; 23-26 May 2004; San Francisco, CA USA. IEEE: New York, USA, 2004, pp60-63.

6 Leemans W, Esarey E. Laser-driven plasma-wave electron accelerators. Phys Today 2009; 62: 44-49.

7 Leemans WP, Duarte R, Esarey E, Fournier S, Geddes CG et al. The BErkeley Lab Laser Accelerator (BELLA): a $10 \mathrm{GeV}$ laser plasma accelerator. AIP Conf Proc 2010; 1299: 3-11.

8 Saraceno CJ, Emaury F, Heckl OH, Baer CR, Hoffmann M et al. 275 W average output power from a femtosecond thin disk oscillator operated in a vacuum environment. Opt Express 2012; 20: 23535-23541.

9 Russbueldt P, Mans T, Weitenberg J, Hoffmann HD, Poprawe R. Compact diodepumped $1.1 \mathrm{~kW}$ Yb:YAG Innoslab femtosecond amplifier. Opt Lett 2010; 35: 4169-4171.

10 Jauregui C, Limpert J, Tünnermann A. High-power fibre lasers. Nat Photonics 2013; 7: 861-867.

11 Eidam T, Hanf S, Seise E, Andersen TV, Gabler T et al. Femtosecond fiber CPA system emitting 830 W average output power. Opt Lett 2010; 35: 94-96.

12 Limpert J, Stutzki F, Jansen F, Otto HJ, Eidam T et al. Yb-doped large-pitch fibres: effective single-mode operation based on higher-order mode delocalisation. Light Sc App/2012; 1: e8, doi:10.1038/Isa.2012.8.

13 Strickland D, Mourou G. Compression of amplified chirped optical pulses. Opt Commun 1985; 56: 219-221.

14 Eidam T, Rothhardt J, Stutzki F, Jansen F, Hädrich S et al. Fiber chirped-pulse amplification system emitting 3.8 GW peak power. Opt Express 2011; 19: 255-260.

15 Fan T. Laser beam combining for high-power, high-radiance sources. Select Top Quantum Electron 2005; 11: 567-577.

16 Augst SJ, Fan TY, Sanchez A. Coherent beam combining and phase noise measurements of ytterbium fiber amplifiers. Opt Lett 2004; 29: 474-476.

17 Seise E, Klenke A, Breitkopf S, Plötner M, Limpert J et al. Coherently combined fiber laser system delivering $120 \mu \mathrm{J}$ femtosecond pulses. Opt Lett 2011; 36: 439-441.

18 Seise E, Klenke A, Breitkopf S, Limpert J, Tünnermann A. 88 W 0.5 mJ femtosecond laser pulses from two coherently combined fiber amplifiers. Opt Lett2011;36:38583860.

19 Zaouter Y, Daniault L, Hanna M, Papadopoulos DN, Morin F et al. Passive coherent combination of two ultrafast rod type fiber chirped pulse amplifiers. Opt Lett 2012; 37: 1460-1462.

20 Bourderionnet J, Bellanger C, Primot J, Brignon A. Collective coherent phase combining of 64 fibers. Opt Express 2011; 19: 17053-17058.

21 Klenke A, Breitkopf S, Kienel M. $530 \mathrm{~W}, 1.3 \mathrm{~mJ}$, four-channel coherently combined femtosecond fiber chirped-pulse amplification system. Opt Lett 2013; 38: 2283 2285.
22 Klenke A, Seise E, Limpert J, Tünnermann A. Basic considerations on coherent combining of ultrashort laser pulses. Opt Express 2011; 19: 25379-25387.

23 Goodno GD, Shih CC, Rothenberg JE. Perturbative analysis of coherent combining efficiency with mismatched lasers. Optics Express 2010; 18: 25403-25414.

24 Mourou G, Brocklesby B, Tajima T, Limpert J. The future is fibre accelerators. Nat Photonics 2013; 7: 258-261.

25 Zhou S, Wise FW, Ouzounov DG. Divided-pulse amplification of ultrashort pulses. Opt Lett 2007; 32: 871-873.

26 Kienel M, Klenke A, Eidam T. Analysis of passively combined divided-pulse amplification as an energy-scaling concept. Opt Lett 2013; 21: 25379-25387.

27 Zaouter Y, Guichard F, Daniault L, Hanna M, Morin F et al. Femtosecond fiber chirpedand divided-pulse amplification system. Opt Lett 2013; 38: 106.

28 Jones RJ, Ye J. Femtosecond pulse amplification by coherent addition in a passive optical cavity. Opt Lett 2002; 27: 1848-1850.

29 Vidne Y, Rosenbluh M, Hansch TW. Pulse picking by phase-coherent additive pulse generation in an external cavity. Opt Lett 2003; 28: 2396-2398.

30 Pupeza I, Eidam T, Rauschenberger J, Bernhardt B, Ozawa A et al. Power scaling of a high-repetition-rate enhancement cavity. Opt Lett 2010; 35: 2052-2054.

31 Carstens H, Lilienfein N, Holzberger S, Jocher C, Eidam T et al. Megawatt-scale average-power ultrashort pulses in an enhancement cavity. Opt Lett 2014; 39: 2595-2598.

32 Pupeza I, Holzberger S, Eidam T, Carstens H, Esser D et al. Compact high-repetitionrate source of coherent $100 \mathrm{eV}$ radiation. Nat Photonics 2013; 7: 608-612.

33 Gohle C, Udem T, Herrmann M, Rauschenberger J, Holzwarth R et al. A frequency comb in the extreme ultraviolet. Nature 2005; 436: 234-237.

34 Jones R, Moll K, Thorpe M, Ye J. Phase-coherent frequency combs in the vacuum ultraviolet via high-harmonic generation inside a femtosecond enhancement cavity. Phys Rev Lett 2005; 94: 193201.

35 Couprie M, Nutarelli D, Roux R, Visentin B, Nahon L et al. Gamma rays produced by intra-cavity inverse Compton scattering of a storage ring free-electron laser. J Phys $B$ 1999; 32: 5657-5667.

36 Börzsönyi A, Chiche R, Cormier E, Flaminio R, Jojart $\mathrm{P}$ et al. External cavity enhancement of picosecond pulses with 28,000 cavity finesse. App/ Opt 2013; 52: 8376-8380.

37 Tünnermann A, Limpert J, Eidam T, Breitkopf S, Pupeza I et al. Methode zur Lichtauskopplung aus optischen Resonatoren mit Hilfe von schnellen, mechanischen Schaltern. German patent DE 102012019 733.0.

38 Britzger M, Wimmer MH, Khalaidovski A, Friedrich D, Kroker S et al. Michelson interferometer with diffractively-coupled arm resonators in second-order Littrow configuration. Opt Express 2012; 20: 25400-25408.

39 Cammarata M, Eybert L, Ewald F, Reichenbach W, Wulff M et al. Chopper system for time resolved experiments with synchrotron radiation. Rev Sci Instrum 2009; 80 015101.

40 Breitkopf S, Klenke A, Gottschall T, Otto HJ, Jauregui C et al. 58 mJ burst comprising ultrashort pulses with homogenous energy level from an $\mathrm{Yb}$-doped fiber amplifier. Opt Lett 2012; 37: 5169.

41 Carstens H, Holzberger S, Kaster J, Weitenberg J, Pervak V et al. Large-mode enhancement cavities. Opt Express 2013; 21: 11606.

This work is licensed under a Creative Commons Attribution-

NonCommercial-NoDerivs 3.0 Unported License. The images or other third party material in this article are included in the article's Creative Commons license, unless indicated otherwise in the credit line; if the material is not included under the Creative Commons license, users will need to obtain permission from the license holder to reproduce the material. To view a copy of this license, visit http://creativecommons.org/licenses/ by-nc-nd/3.0/ 